\title{
Application Research of Extraction Algorithm of Image Feature Information in Intelligent Video Monitor
}

\author{
Haoyin Lv \\ School of Information Engineering, Longdong University, Qingyang, 745000, China
}

Keywords: Extraction algorithm, Image feature information, Intelligent video monitor

\begin{abstract}
With the help of the powerful data processing function of the computer, the intelligent video monitoring can analyze the massive data and extract the valuable information of the video. The extraction algorithm of image feature information in intelligent video surveillance is a hot and difficult problem in the field of image processing. This paper analyzes the differential structure of image, the basic flow, the algorithm principle and the algorithm connotation of image feature extraction in intelligent video to provide some references for the related researchers.
\end{abstract}

\section{Introduction}

With the rapid development of economy and the progress of urbanization, emergencies and unusual events in production and life are becoming more and more complex. The difficulty and importance of monitoring is becoming more and more prominent. The traditional monitoring method is more and more prominent. Intelligent video analysis and monitoring is a new product born under this market demand. Its development is inseparable from the support and development of many hardware and software technologies. If the camera as the human eye, so the intelligent video analysis system can be regarded as the brain intelligent video surveillance system were realized from the visual interpretation of changes to the automatic interpretation, this is the biggest difference between the intelligent video surveillance system and common monitoring system, and the inevitable trend of the development of video surveillance technology. Its advantage lies in the continuous automatic analysis and processing of video data. The implementation of intelligent video surveillance technology is inseparable from webcam, data storage computing equipment and data transmission equipment. Webcam has independent network card, video compression card and micro operation system. It can directly connect to the network and build many intelligent functions, such as server, alarm management, digital video compression, and programmable two developments. The latest webcam products have the advantage of supporting power supply, and there is no need to lay a power line for the camera. The cable of analog monitoring camera cannot satisfy long distance transmission, while the network cable of the network camera is a network cable. When long-distance transmission is needed, optical fiber transmission can be used. The optical fiber not only has strong anti-interference ability, but also has lower signal attenuation and higher security in the transmission process. There are three kinds of intelligent video analysis technology: target detection technology, target tracking technology and behavior recognition technology. Object detection is to process real-time information collected by various visual analysis algorithms, including image pre-processing, image segmentation, feature extraction, filtering candidate color blocks, and ultimately extract a region of interest.

\section{Analysis of Image Differential Structure}

\subsection{Normalization Treatment.}

The visual perception of the image depends on the visual condition, such as the condition of the visual distance. Therefore, the image should be normalized to a certain visual condition before 
extracting the image information. In this paper, the image appearance model is used to normalize the image. If the visual condition changes, the distortion below the threshold may be converted to a distortion higher than the threshold. The image appearance model is still in the development stage. For example, the contrast sensitivity mechanism is usually modeled as a convolution in intensity linear relative color space, and different filters are applied to color and non-colored channels in frequency domain. This involves several simplification processes. Because the sensitivity mechanism is directional, age, and influenced by light conditions. The other components of the image appearance model are also restricted by these conditions. This paper shows whether a simple image appearance model can improve the performance of the image differential prediction. In this paper, an image feature extraction algorithm for image segmentation using feature recognition technology is proposed in the process of deep image acquisition. The collected images are processed by smoothing, two value and image segmentation. After that, the extracted files are reasonably compressed to extract effective data, which will remove the larger part of subsequent data processing. It solves the problem that information is not easy to be removed in image information, realizes data parallel processing, reduces manual interference in measurement process, greatly accelerates measurement speed, and provides basis for subsequent data processing and feature recognition. Finally, the method is verified by the test.

\subsection{Color Conversion Processing.}

In the final phase of normalization, all images need to be converted to the same color space. This color space needs to provide information, brightness, chromaticity, color, and so on for all the factors that affect color. One of the most important features is perceptual consistency. The distance between the color space should match the difference of the perceptual color. This requires an accurate representation of the features of the image, such as edges and gradients. In the RGB color space, these features may be overestimated or underestimated, that is, the magnitude of the calculation exceeds the amplitude of the perception or the amplitude of the perception. Don't place a background image acquisition object, and then under the same conditions, collecting placed measurement image objects to be measured with the simple contrast, then use the subtraction method for the measurement of image background subtraction image can get the image of the measured object. Get the image processing color of the object in front of a picture in addition to the analyte in gray value close to zero, the value of difference and the object image to be measured. First use the average template to smooth the image, and then the application value domain binarization procedure of image gray parameter setting value, then after processing color image. This will bring some difficulties to the subsequent processing of target recognition. The sub pixel edge detection operator extracts the image edge effect obviously, get the target object contour more accurately, can achieve better noise removal effect. In a program called feature extraction operator edge image color processing sub pixel edge detection before, get the edge depth image of the measured object.

\subsection{Image Segmentation Processing.}

In the use of the structured light three-dimensional data acquisition technology, with the depth image of structured light stripe is to realize the task of measuring the source of information, it includes the measurement of shape information of the object surface, processing and calculation of the structure of the optical depth image is one of the key aspects of the measurement data of the task. The difficulty of data extraction is greatly increased by many environment and various forms of noise interference in the depth image. How to quickly and accurately remove these interferences and extract the structural light fringe data in the image is the key to the processing and calculation of the structure light depth image. There are many ways to remove noise interference, in which the image segmentation method can fulfill the requirement of noise removal better. Most of the research work on image segmentation is focused on how to get an optimal segmentation result. However, in practical applications often require different restraint for example, multiple segmentation results under different threshold, this is in the required information is often scattered in many different grades, under the same constraints cannot be extracted. It is a time-consuming and frustrating method 
to run a segmentation algorithm to run many times under different constraints to meet the requirements above. The image feature information extraction algorithm is proposed for the problem of noise removal and data compression in the process of image acquisition. The characteristics of image recognition technology commonly used in the treatment of introduced into the image acquisition process, a series of images of the measuring equipment according to the order of acquisition of the subtraction operation, threshold processing transform, edge extraction, image segmentation, image acquisition method of regional dynamic domain area effective display and segmentation in image acquisition and pre-acquisition. Depth image includes useful information, including environmental information and other useless information. Useful information and useless information divide the deep image into two parts, and the two part has obvious boundaries.

\section{Extraction Algorithm of Image Feature Information in Intelligent Video Monitor}

\subsection{Basic Flow}

The realization of video analysis function cannot be separated from the support of computer vision algorithm. By studying the principles of various basic visual algorithms, according to their different characteristics and application scope, according to certain rules, we design the algorithm process, and we can achieve specific purpose monitoring tasks. The research of video analysis algorithms will be introduced in detail later, including how to apply them to the analysis and extraction of scene information in manufacturing service process. According to user's intelligent analysis and control time interval and time interval, we get a video data acquisition and analysis for the current state scene every occasionally. When the end of the analysis, the processing result of the record to file state data table monitoring point database corresponding to the day after, convenient check and statistical analysis. The object of analysis of surveillance video cloud manufacturing service process is collected from each scene video stream, before we make an analysis on the video stream, need access to video data. The camera development package provides a callback interface for data streams. Get video data from the camera. The measurement data part that needs to be collected is needed in the measurement. Image acquisition region segmentation can eliminate all noise interference except the object being measured. The feature edge divides the whole image into two parts, and uses this feature to assign the gray value of the image outside the edge curve to black in the process of image acquisition. The line structure light is intercepted by the characteristic edge, and it is the part of the measurement data that needs to be collected in the measurement. At this point, the image is clear and contains all the information needed to extract the data, which does not affect the accuracy of the data extraction. The data structure is simplified and it is convenient for data extraction.

\subsection{Algorithm Principles}

The image local preprocessing method is smooth, also called image blurring, and it is used in digital image processing to reduce and suppress the image noise. Image smoothing technology is not very complicated and the principle is very simple, but the use of cards in image processing is one of the basic algorithms that we must master. This is the simplest filter at present. Because we only need to calculate the average number of the coverage area around each pixel value of the core error, we can get the pixel value after output. This paper does not use this method to filter, and does not make a detailed introduction here. The idea of this filtering algorithm is also very simple. It mainly uses the huge difference between the pixel value of noise point and other pixels, which will make noise points arranged at the two ends of a sequence in a small piece of kernel coverage according to pixel size. Therefore, taking the median as the output pixel value will filter out the noise point. The kernel of Gauss filter is the weight coefficient distribution that conforms to the normal distribution. The point in the image needs to be convolution with the Gauss kernel to beam out the output pixel value. It is probably the most useful filter at present. Although it can process slower than other filters, it can get comparable results in general image noise filtering. Like Gauss filtering, bilateral filtering is also a kernel structure that constructs a weight coefficient distribution. When sliding to a pixel location, the 
weighted average of its neighborhood is obtained. It is different from Gauss weighting. It is the difference between point and melon pixels as the basis for determining weight coefficients, rather than the distance between two points. Similar pixel values will give higher weight coefficients. Edge detection and contour extraction are widely used in image segmentation, target classification, image matching and pattern recognition. These applications are the basic research of intelligent video analysis technology.

\subsection{Algorithm Analysis}

Color feature is the most widely used visual feature in image retrieval. The main reason is that color is often related to objects or scenes contained in images. Compared with other visual features, color features are less dependent on the size, direction, and angle of view of the image itself, and thus have high robustness. In the field of computer vision, can using color image pixel values of the channel ratio or difference, or some other rules to certain colors were selected to filter out some of the target color pixel value differences, to achieve the purpose of reducing the amount of data calculation. The indicator color of machine tool is one of the most important characteristics to evaluate its running state. It is possible to judge the basic state of machine tool based on indicative and clever color, but it is impossible to get the accurate operation parameters of machine tool by sensors. Production and processing process is the core link of manufacturing service as well as one of the key points of monitoring. It is envisaged that in the future, as the number of service providers entering the cloud manufacturing platform is increasing, the size of the resource pool will also increase, providing more kinds of manufacturing services and stronger service capabilities. Then the number and distribution of the monitoring points corresponding to it will be further expanded. How to maintain and manage thousands of devices remotely is a vital function for the platform. For video collection devices distributed on various monitoring sites, equipment resource repository can be built through equipment virtualization technology to facilitate centralized management of platforms. Different orders execution process needs to be bound to the corresponding regional control devices by platform. In this way, we can not only achieve dynamic monitoring of equipment resource scheduling, but also detect the running status of various devices in real time, and locate and maintain the fault equipment in time. The purpose of video surveillance for various production and processing sites is mainly to understand workers' working status and equipment running status. The indicator light is relative to the surrounding background brightness and saturation are relatively high, the design algorithm considering RGB color space representation of the image is converted to HSV color space, filtering work candidate targets by color brightness and saturation information, can improve the recognition accuracy and color judgment.

\section{Conclusions}

As monitoring video is gradually moving towards high-definition, future data storage will become a key issue. The latest cloud storage technology and it can solve this problem well, save a lot of monitoring video data, which can be accessed later, and can also enhance data security and data backup and restore function. It is believed that in the future, the remote monitoring system will be able to meet all kinds of scenes' video information collection and automatic analysis and processing in mass distributed manufacturing environment, and truly realize the purpose of intelligent monitoring.

\section{References}

[1] Jiang Jian, Tang Yongming. Design of the Video Frame Information Detection and OSD [J]. Chinese Journal of Electron Devices, 2014, 37(5): 835-839.

[2] Yang Dan. Color Image Difference Prediction Based on Image Difference Measure [J]. Computer Science, 2015, 42(1): 308-311+316. 
[3] Du Guangchao, Liu Xiao, Shao Mingsheng. Image contour feature information extraction based on genes encoding algorithm [J]. Laser \& Infrared, 2013, 43(7): 818-821.

[4] Rao Ping, Wang Jianli, Wang Yong. Extraction of information on construction land based on multi-feature decision tree classification [J]. Transactions of the Chinese Society of Agricultural Engineering (Transactions of the CSAE), 2014, 30(12): 233-240. 